\title{
Socio-économie de la pêche artisanale dans les eaux burundaises du lac Tanganyika à Mvugo et Muguruka
}

\author{
Gabriel MUKABO OKITO ${ }^{*}$ Jean-Claude MICHA ${ }^{2}$, Jean BOKASSA HABARUGIRA ${ }^{3}$, \\ Gaspard NTAKIMAZI ${ }^{4}$, Venant NSHOMBO MUDERHWA ${ }^{5}$, \\ Phanuel BIZURU NZIBONERA ${ }^{6}$ et B. Georges MUHIRWA ${ }^{6}$
}

\author{
${ }^{1}$ Maître en Science, Assistant à la section Eaux et Forêts de l'Institut Supérieur Agrovétérinaire et \\ Conservation de la nature de Minembwe (ISAVC/Minembwe/RDC), BP-6295 Bujumbura, Burundi. \\ ${ }^{2}$ Professeur Emérite au Département de Biologie, Unité de Recherche en Biologie Environnementale (URBE), \\ Université de Namur, BP-5000 Namur, Belgique. \\ ${ }^{3}$ Maître en Science, Assistant au Département de Biologie, Université du Burundi, BP-2700 Bujumbura, \\ Burundi. \\ ${ }^{4}$ Professeur Ordinaire, Faculté des Sciences, Université du Burundi, BP-2700 Bujumbura, Burundi. \\ ${ }^{5}$ Professeur et Maître de Recherche au Département de Biologie, Centre de Recherche en Hydrobiologie \\ (CRH/Uvira) BP-73 Uvira, R.D. Congo. \\ ${ }^{6}$ Section Agrovétérinaire, Institut Supérieur Agrovétérinaire et de Conservation de la nature de Minembwe \\ (ISAVC/Minembwe, RDC), B.P. 6295 Bujumbura, Burundi. \\ *Auteur correspondant ; E-mail : mgokito@gmail.com ; Tél. +24382 1270424
}

\section{REMERCIEMENTS}

Les auteurs demeurent reconnaissants à l'Université du Burundi et à la Commission Universitaire du Développement (CUD) de Wallonie-Bruxelles pour le partenariat grâce auquel une partie du travail de terrain a été financée.

\section{RESUME}

Une étude sur la dynamique socio-économique et environnementale de la pêche artisanale au Burundi a été effectuée dans le nord-ouest du Lac Tanganyika, coté burundais, dans la province de Makamba, Commune de Nyanza-lac, aux sites de débarquement (plages) de Muguruka et Mvugo entre mars et juillet 2013. Les objectifs étaient d'actualiser l'information sur les pêcheurs, leurs associations, les engins utilisés et l'évolution des prises en vue de la comparer avec des données plus anciennes, et d'analyser les coûts et revenus des unités actives, motorisées ou non motorisées, afin de préciser leur rentabilité. Dans l'ensemble, 130 personnes ont été interrogées et 6400 poissons ont été mesurés. Stolothrissa tanganicae (adultes et juvéniles) et Lates stappersii juvéniles ont dominé dans les captures avec respectivement $68 \%$ et 26,7\% à Muguruka, $81,5 \%$ et $16,7 \%$ à Mvugo. L'étude estime la capture par unité d'effort de l'unité Appolo à $152 \mathrm{~kg} / \mathrm{nuit} \mathrm{de}$ pêche, $80 \mathrm{~kg} /$ nuit de pêche pour le catamaran motorisé et $50 \mathrm{~kg}$ /nuit pour le catamaran non motorisé. Après la vente des produits de la pêche, le profit net estimé (PNE) par unité de pêche artisanale s'élève à $8300 \pm 222$ \$US. avec un revenu annuel net par pêcheur d'environ 691 \$US pour le catamaran non motorisé et de $4380 \pm$ 654 \$US, avec un revenu annuel net par pêcheur de 274 \$US pour le catamaran motorisé ; tandis que pour l'unité Appolo, le PNE s'élève à $11670 \pm 2500$ \$US avec un coût salarial par pêcheur de 645 \$US. On constate que les unités non motorisées sont plus rentables dans la zone proche des rives. Quant aux unités Apollo pêchant au large en pleine zone pélagique, les captures sont nettement plus élevées mais leur rentabilité n'est guère supérieure aux unités non motorisées.

(C) 2017 International Formulae Group. All rights reserved.

Mots clés : Capture, Clupéidés, effort de pêche, investissement, rentabilité. 


\title{
Socio-economy of the small-scale fishing in the burundian waters of lake Tanganika at Mvugo and Muguruka
}

\begin{abstract}
The study on the socioeconomic and environmental dynamics of the small-scale fishing in Burundi was made in the Northwest part of the Lake Tanganyika, quoted burundian, in the province of Makamba, Municipality of Nyanza-lake, in the sites of landing of Muguruka and Mvugo during the period from March till July, 2013. It has for objective, on one hand, to update the information about the fishermen, their associations, the machines used in both selected beaches as well as evolution of the grips to compare her with more former data, and analyze the costs and the income of the active, motorized and not motorized units, to specify their profitability. Stolothrissa tanganicae (grown-up and young) and young Lates stappersii dominated in captures with respectively $68 \%$ and $26.7 \%$ to Muguruka, $81.5 \%$ and $16.7 \%$ to Mvugo. As for the capture by unit of effort (CPUE), the study estimates the CPUE of the unit Appolo at $152 \mathrm{~kg} / \mathrm{night}$ of peach, $80 \mathrm{~kg} / \mathrm{night}$ of peach for the motorized catamaran and $50 \mathrm{~kg} /$ night for the not motorized catamaran. After-sales service of the products of peach(fishing), the Clear(Net) Profit Considered (PNE) by unit of small-scale fishing amounts to $\$ 8300 \pm 222$ US with a clear (net) annual income by fisherman about 691 US for the not motorized catamaran, there are $4380 \$ \pm 654 \mathrm{US}$, with an income.
\end{abstract}

(C) 2016 International Formulae Group. All rights reserved.

Keywords: Catch, fishing effort, investment, profitability, Clupeids, Tanganyika

\section{INTRODUCTION}

Situé entre le Burundi, la République Démocratique du Congo, la Tanzanie, et la Zambie, le Lac Tanganyika est très réputé pour le nombre important d'espèces endémiques. On note pas moins de 250 espèces de poissons Cichlidés et 150 espèces de non Cichlidés dont la plupart vivent le long de la côte jusqu'à environ 180 mètres de profondeur (Sarvalaet al., 2006). Toutefois, la plus grande part de la biomasse se situe dans la zone pélagique et est dominée par six espèces: deux espèces de Clupéidés (sardines) endémiques du Tanganyika: Stolothrissa tanganicae (Regan, 1917) et Limnothrissa miodon (Boulenger, 1906 ; Roest, 1978, 1992, 2014; Mannini et Aro, 1995; Mölsä et al., 1999) et quatre espèces de Latidae, endémiques également, dont principalement Lates stappersii (Boulenger, 1914). La quasi-totalité des espèces de Cichlidés est aussi endémique et plusieurs sont appréciées comme poissons d'aquarium (Van der Knaap et al., 2014).

Plusieurs menaces pèsent sur l'écosystème du Lac Tanganyika et sur sa richesse biologique. La non-application des lois sur la pêche ainsi que la croissance démographique et de plus de $2 \%$ par an (PNUD, 2013) que connaissent les pays riverains du Lac Tanganyika en général et le Burundi en particulier, exercent une pression croissante sur les ressources halieutiques du lac en effectuant des prélèvements de plus en plus conséquents pour satisfaire ses besoins nutritionnels sans aucune considération pour les stocks exploités. Ceci conduit non seulement à la destruction des écosystèmes aquatiques, mais également à l'extinction de certaines espèces (Bongeba et Micha, 2013).

Les clupéidés à eux seuls comptent pour environ $80 \%$ du poids de la capture totale annuelle (Coenen et al., 1998) évaluée à quelques $250.000 \mathrm{t} / \mathrm{an}$. Vu leur importance commerciale considérable, de nombreux chercheurs se sont intéressés à l'étude de leur biologie en vue de générer les connaissances nécessaires pour l'aménagement et le développement de la pêche dans le lac Tanganyika (Mulimbwa, 1995).

Cette étude analyse la dynamique socio-économique et environnementale de la 
pêche artisanale dans le Lac Tanganyika au Burundi et vise à :

$\checkmark$ Actualiser l'information sur les pêcheurs, leurs associations, ainsi que sur les engins utilisés aux deux plages sélectionnées en vue de la comparer avec des données plus anciennes ;

$\checkmark$ Observer la mobilité des pêcheurs et l'évolution des prises par rapport à l'enquête cadre de 2011 ;

$\checkmark$ Analyser les coûts et revenus des unités actives, motorisées et non motorisées, afin de préciser leur rentabilité.

\section{MATERIEL ET METHODES Milieu d'étude}

La présente étude a été effectuée en République du Burundi au sud-ouest de la province de Makamba, dans la commune de Nyanza-lac à environ 100 et $115 \mathrm{~km}$ au sud de Bujumbura sur la RN3, précisément aux sites de débarquement (plages) de Muguruka et Mvugo, coordonnées $03^{\circ} 28^{\prime} 34.4^{\prime \prime}$ latitude Sud et $29^{\circ} 32^{\prime}$ '44" longitude Est. Le choix de ces deux sites est justifié d'une part, par le nombre élevé des unités des pêches qui y débarquent par rapport à d'autres sites du pays et d'autre part, par le fait que la plus grande quantité de poissons vendus au Burundi proviennent de ces sites.

\section{Méthodes de collecte des données}

Les données ont été collectées auprès des unités de débarquement de mars à juillet 2013 (5 mois), à raison de 4 sorties par mois, de 6 à 8 heures du matin, aux 2 sites de débarquement sélectionnés (Muguruka et Mvugo). Les poissons ont été prélevés au hasard dans les captures débarquées directement du lac.

Dans cette étude, nous avons recouru à deux méthodes. La méthode empirique appuyée par les recherches documentaires relatives au thème de recherche et les entretiens avec les personnes ressources.

L'observation a constitué un élément déterminant de notre dispositif méthodologique. En effet, les moments d'observation ont été mis à profit pour notamment identifier (Poll et Gosse, 1995) non seulement les différents genres et espèces de poissons capturés durant notre étude, mais aussi les engins de pêche utilisés et surtout clarifier certaines réalités de la pêche que nous n'avions pas saisies durant les entretiens organisés.

Les entretiens semi-structurés réalisés avec des pêcheurs réunis en « focus group » ont permis de répondre à quelques questions pour lesquelles il n'est pas aisé d'obtenir des réponses concises par questionnaire. Il s'agissait entre autres du mode d'exploitation de la pêche, de sa gestion, de la conservation des poissons, de l'organisation sociale et de la division du travail selon lesexe.

Nous avons aussi recouru à la méthode quantitative soutenue par la technique d'enquêtepar sondage à choix raisonné (Grawitz in Luhusa et Micha, 2013), car celleci permet de menerune étude sur une partie de la population qui a les mêmes caractéristiques ou qui exerce les mêmesactivités. Les participants aux focus-groupes étaient systématiquement tirés de manière aléatoire sur base de représentation des différents acteurs intervenant dans le secteur de la pêche à savoir: le chef de plage, un membre du comité de la Fédération des Pêcheurs Fournisseurs de Poissons au Burundi, un agent du département de pêche et pisciculture du Burundi affecté sur la plage, le président du comité de pêcheurs de la plage, le représentant des chefs d'équipage des unités de pêche, un représentant des propriétaires d'unité de pêche, deux pêcheurs et trois représentants des associations des pêcheurs.

Sur une population de 376 personnes actives à Muguruka, 40 individus ont aléatoirement été sélectionnés pour autant qu'ils pratiquent la pêche. 90 personnes sur un total de 810 inventoriées à Mvugo ont été choisies pour répondre à nos questionnaires.

Le nombre d'unités débarquant, les Captures (kg) par Unité d'Effort de Pêche (CPUE: 1 
nuit de pêche par catamaran) et le prix de vente des poissons sur la plage ont été enregistrés. Les statistiques quotidiennes de Captures par Unité d'Effort de pêches (CPUE) nous ont été fournies par un enquêteur affecté à chaque site par la Direction des Pêches. Sur le terrain, à part la planche graduée au $\mathrm{mm}$ et la balance précise à $0,1 \mathrm{~g}$, nous avons utilisé un appareil photo numérique et un GPS.

\section{Traitement de l'échantillon}

A l'aide d'une planche graduée, les longueurs totales (LT en $\mathrm{mm}$ ) étaient mesurées pour tous les individus de chaque échantillon.

\section{Analyse de coûts et revenus des pêcheurs}

Pour arriver à déduire la marge bénéficiaire d'une unité artisanale dans le site étudié et en sortir le revenu net par artisan pêcheur, quelques indicateurs économiques ont été calculés à partir d'une unité type (Njifonjou, 2010) Ces indicateurs sont: Capital Investi (CI): Somme d'achat de tous les matériels constituant l'unité de pêche, Coût Opérationnel (CO): Ensemble de toutes les dépenses, Coût d'Exploitation (CE) : $C O$ Amortissement, Profit brut estimé (PBE) : total des revenus obtenus après-vente, Profit net estimé (PNE) : Marge bénéficiaire de l'unité de pêche (PBE - CE), Taux de rentabilité (TR): Indice permettant de calculer le délai de récupération du capital investi (PNE/C.I *100)et Coût salarial (CS): = revenu par pêcheur.

\section{Analyse statistique}

Les tableaux synthèses des données, la statistique descriptive (moyennes, écart-types, etc.) et la construction des graphiques ont été réalisés à l'aide du logiciel Microsoft Excel (version 2010) sous Microsoft Windows 7.

Pour les analyses statistiques, le logiciel SPSS 14.0 sous Microsoft Windows 7 a été utilisé. L'ANOVA One-Way a été utilisée pour la comparaison des moyennes de différentes tailles des différentes espèces de poissons capturées.

\section{RESULTATS \\ Typologie de la pêche et évolution des Unités artisanales}

La pêche est surtout pratiquée la nuit car la majorité des méthodes utilisées se basent sur l'attraction des clupéidés par la lumière ; qu'il s'agisse de la pêche au carrelet, à la senne de plage ou au filet encerclant. C'est la raison pour laquelle, les activités de pêche cessent pratiquement chaque mois durant la pleine lune (environ 10 jours). On distingue deux types d'unités de pêche artisanale bien identifiables sur le Lac Tanganyika en générale et les sites de Muguruka et Mvugo en particulier : l'unité catamaran (motorisé et non motorisé) et l'unité Appolo. La pêche artisanale emploie plus de $80 \%$ des personnes actives dans la filière de pêche à Muguruka comme à Mvugo (Figures 2 et 3). Le nombre total de pêcheurs actif opérant à Muguruka est estimé à 432 , pour environ 54 embarcations, faiblement motorisées (41\%). Il est de 936 à Mvugo pour 117 embarcations parmi lesquelles 49\% seulement sont motorisées (Tableaux 1 et 2).

En plus des filets carrelet utilisés par les unités de pêche artisanale débarquant le long du Lac Tanganyika, coté burundais en général et les plages de Muguruka et Mvugo en particulier, on a aussi inventorié un bon nombre de filets maillants et de sennes de plage utilisés par les mêmes unités artisanales. (Tableau 3).

En ce qui concerne les unités de pêche artisanales, la Figure 2 montre que le site de Mvugo présente plus d'unités de pêche artisanale que le site de Muguruka. Par rapport à l'évolution, les résultats de la même figure montrent qu'en général, le nombre d'unités reste stable (Muguruka) ou ne fait que baisser (Mvugo).

Les résultats du tableau 1 montrent qu'à Muguruka, par rapport à l'enquête cadre de 2011, les unités catamaran non motorisé augmentent de 50 à $77 \%$. Par contre, les unités catamaran motorisé et les unités Appolo ont sensiblement baissé avec un taux de réduction respectif de $36,4 \%$ et $34,8 \%$. 
Il ressort du tableau 2, que le taux d'augmentation des unités catamaran non motorisé est de l'ordre de 29,7 à $62,2 \%$ tandis qu'il est de 66,7 à $133,3 \%$ pour les catamarans motorisés mais leur nombre reste très faible. Quant aux unités Appolo, ces résultats montrent une réduction de l'ordre de 36, 9 (2012) à 54,9\% (2013).

Il ressort du tableau 3 que la senne de plage vient en tête avec $42,9 \%$ suivi du filet carrelet avec $35,9 \%$ puis du filet maillant avec $21,3 \%$. Quant à la taille de maille des filets utilisés, on note l'abondance de filets à petites mailles, inférieures à $5 \mathrm{~mm}$, qui représentent $65,3 \%$. Les filets de maille égale ou supérieure à $5 \mathrm{~mm}$ ne représentent que $24,7 \%$.

Les figures 3 et 4 montrent que les artisans pêcheurs (propriétaires et membres d'équipage) ont un âge généralement entre 18 et 40 ans (67\%), parmi eux, $69 \%$ sont mariés avec un niveau d'instruction très faible, et plus de $80 \%$ des personnes inventoriées sont engagées dans la pêche comme occupation principale mais elles ont aussi une autre activité de subsistance comme l'agriculture et la commercialisation de produits divers. Tous les pêcheurs font partie d'associations regroupées au sein de la Fédération des Pêcheurs et Fournisseurs de poissons au Burundi et leurs problèmes majeurs sont constitués essentiellement par les faibles captures et le prix élevé du carburant et des engins de pêche.

\section{Suivi des Captures, transformation et commercialisation}

Lors des débarquements matinaux aux deux plages, les Captures journalières Par Unité d'Effort (C.P.U.E.) ont été mesurées en poids $(\mathrm{kg})$ par unité de pêche. La capture moyenne journalière par catamaran non motorisé est de $50 \mathrm{~kg}$ par nuit. Ceci est particulièrement faible si on considère que chaque unité à un équipage de 6 à 8 personnes et que chaque pêcheur a en moyenne 8 à 9 personnes à charge, cela représente moins de $695 \mathrm{~g}$ par personne et par jour. Les autres unités de pêche artisanale (motorisée et
Appolo) semblent plus efficaces en terme de production $(80 \mathrm{~kg} /$ nuit pour le catamaran motorisé et $152 \mathrm{~kg}$ pour l'unité Appolo), mais si on considère d'un côté les dépenses effectuées en terme d'intrant et d'autre part l'équipage de 6 à 8 personnes plus les personnes à charge, on arrive à des chiffres similaires ou moins à celui obtenu pour l'unité catamaran non motorisée. En ce qui concerne la production des unités de pêche artisanale, notre étude renseigne que les captures débarquées à Muguruka proviennent des unités Appolo $(44,6 \%$, ) des unités catamaran motorisé $(35,5 \%)$ puis des unités catamaran non motorisé $(19,9 \%$.). La capture des unités Appolo domine aussi les autres unités de pêche artisanale à Mvugo avec 54,4\%, les catamarans motorisés viennent en deuxième position avec $40 \%$ et les catamarans non motorisés ne représentent que 5,6\%. Comparés aux résultats de PRODAP 2011, on remarque toutefois une réduction de capture d'environ $20,5 \%$ et $36,7 \%$ respectivement à Muguruka et à Mvugo (Figures 5 et 6). En général, au retour d'une pêche collective au lamparo (senne tournante ou carrelet), lepatron de pêche sépare la prise en fonction du coût du matériel (essence, pétrole) et divise le reste entre lui et les pêcheurs. On observe des corrélations significatives entre le nombre d'unités de pêche, les engins utiliséset les captures (Tableau 4) : plus il y a d'unités $(r=$ $0,890)$, plus il y a augmentation du nombre de lampes à utiliser $(r=0,895)$ sans qu'il y ait augmentation de la capture $(\mathrm{r}=-0,35)$.

La transformation du poisson se fait de manière artisanale et assez rudimentaire. Les clupéidés, principales espèces concernées par cette méthode, sont séchés à même le sable. Dans certains cas, le poisson est séché sur des claies, ce qui apprécie la valeur du produit fini d'environ $25 \%$ et diminue les pertes par brisure. Le séchage du poisson rencontre également de sérieux problèmes en période de pluies, en raison des conditions de séchage difficiles et du manque de magasins de stockage à Muguruka. Le fumage concerne surtout les Lates stappersii (mukeke) et les 
techniques utilisées sont rudimentaires et consommatrices de bois. Le poisson est vendu à l'état frais surtout dans les zones situées près des centres de consommation. Mvugo, approvisionné en électricité, présente des infrastructures acceptables pour le frais. En cas d'abondance, les Lates stappersii (mukeke) y sont congelés par les comités de pêcheurs regroupés au sein de leur association AFPOMABU, avant d'être distribués vers les zones à fort pouvoir d'achat, comme la ville de Bujumbura.

Les autres acteurs de la filière sont au nombre de 105 à Muguruka et 128 à Mvugo, dont respectivement $44 \%$ et $40 \%$ de femmes actives dans la transformation et la commercialisation. À l'arrivée des pêcheurs, les vendeurs, généralement des femmes, sillonnent les plages pour acheter les poissons auxpêcheurs, un vendeur a 2 ou 3 pêcheurs attitrés. En général, le poisson est vendu directement sur les marchés ou au bord de la route sans transformation. En cas de surplus, les sardines (nadgala» (Stolothrissa et Limnothrissa) et petits "Mukeke » (Lates stappersii) sont séchés et les grands « Mukeke » sont fumés (Photo 2). Les poissons sont vendus par petits paquetsou au $\mathrm{kg}$. Il existe une forte concurrence entre les vendeurs en raison de la raréfaction des poissons. Lorsque les captures sont vraiment faibles, les pêcheurs préfèrent vendre le peu de poissons qu'ils ont, afin de pouvoir acheter de la farine de manioc ou autres nourritures plus " substantielles » pour eux et les personnes qu'ils ont à charge.

Les pirogues de pêche traditionnelle et artisanale sont fabriquées localement par des charpentiers, qui disposent d'un savoir-faire avéré en la matière.

Considérant les captures par unité de pêche artisanale, les résultats des Figures 5 et 6 révèlent que pour la totalité des poissons débarqués à Muguruka comme à Mvugo en 2013, les unités Appolo occupent la première place suivies des unités catamarans motorisées et enfin viennent les unités de pêche artisanale catamaran non motorisées avec respectivement 17,5 tonnes $(44,8 \%), 13,85$ tonnes $(35,3 \%)$ et 7,8 tonnes $(19,9 \%)$ de la capture débarquée à la plage de Muguruka contre 60,8 tonnes $(56,3 \%), 44,5$ tonnes $(41,2 \%)$ et 2,6 tonnes $(2,5 \%)$ de la capture débarquée (Photo 1) à la plage de Mvugo.

La Figure 7 permet de mettre en évidence que, pendant la période d'échantillonnage, Stolothrissa tanganicae (adultes et juvéniles) et Lates stappersii juvéniles ont dominé dans les captures avec respectivement $68 \%$ et $26.7 \%$ à Muguruka contre $81,5 \%$ et $16,7 \%$ à Mvugoet cela pour toutes les unités de pêche artisanale confondues. Les autres catégories, $L$. stappersii adultes et Limnothrissa miodon étaient moins représentées dans les captures tout au long de notre enquête.

Un total de 2.850 spécimens a fait l'objet de mensurations à Mugaruka, soit 1.225 (43\%) Stolothrissa tanganicae, 1.054 (37\%) spécimens de Limnothrissamiodon et $571(20 \%)$ spécimens de Latesstappersii (juvéniles et adultes). A Mvugo 3.550 spécimens ont fait l'objet de mensurations dont $1.260(35,5 \%)$ Stolothrissa tanganicae, $1.096(30,9 \%)$ Limnothrissa miodon et 1.194 (33,6\%) Lates stappersii (juvéniles et adultes). La plupart des individus de Stolothrissa tanganicae et Limnothrissa miodon étaient matures tandis que la majorité des Lates stappersii étaient immatures. Les résultats $\mathrm{du}$ tableau 6 montrent que les tailles moyennes de $L$. miodon, $S$. tanganicae et $L$. stappersii sont quasi semblables à Muguruka et Mvugo.

Les résultats du Tableau 5 montrent que, sur l'ensemble des deux sites, $45,7 \%$ de la capture sont réalisés par les filets à mailles de moins de $4 \mathrm{~mm}$. Les juvéniles de Stolothrissa tanganicae dominent avec $62,5 \%$ suivi des juvéniles de Lates puis des Limnothrissa miodon avec respectivement $24,5 \%$ et $9,2 \%$.

La Figure 8 montre que le site de Mvugo dispose de plus de $70 \%$ des infrastructures d'appui à la pêche alors que le site de Muguruka en dispose moins $( \pm 25 \%)$.

La Figure 9 montre que la pêche occupe plus de $80 \%$ des personnes dans les 
deux sites, viennent ensuite les mareyeurs et les transformateurs (séchage et fumage des poissons).

\section{Rentabilité économique des unités de pêche artisanales}

Dans cette section, nous présentons la quantité et les différentes puissances des moteurs utilisés par site (Figure 10), le nombre moyen des lampes utilisées par unité et par site (Tableau 6), les indices de performance économique (en \$US) des unités de pêche artisanale de la côte sud burundaise du Lac Tanganyika en 2013 (Tableau 7).

\section{Engins utilisés}

La pêche artisanale au Lac Tanganyika comprend deux types d'unités spécialisées dans la pêche aux clupéidés et aux Lates stappersii de petite taille à savoir le catamaran (à rame et motorisé) et le trimaran ou Appolo.

- Catamaran à rame (non motorisé)

L'unité catamaran non motorisé se compose de 2 pirogues en planches de 6 à $7 \mathrm{~m}$, reliée entre elles par des perches, équipée autrefois de 6 à 8 lampes (actuellement 10) et propulsées par les rames. Elle est équipée d'un filet carrelet (lift net) artisanale classique, que l'on rencontre au Burundi, en Tanzanie et en R D Congo.Le filet carrelet fait généralement $70 \mathrm{~m}$ de circonférence pour $15 \mathrm{~m}$ de chute. L'ensemble d'unité catamaran à rame coûte environ 1700\$US (soit 3230000 $\mathrm{FBu}$ ). Elle emploie entre 4 et 5 pêcheurs.

- Catamaran motorisé

L'unité catamaran motorisé se compose aussi de 2 pirogues en planches de 6 à $7 \mathrm{~m}$, reliée entre elles par des perches, équipée autrefois de 6 à 8 lampes (actuellement 12) et propulsées pardes moteurs de 15 à $25 \mathrm{CV}$. Elle est équipée aussi d'un filet carrelet (lift net) artisanal classique de même circonférence que celui utilisé par le catamaran non motorisé. L'ensemble de l'unité catamaran motorisé coûte environ 3.600\$US (soit $6840000 \mathrm{FBu}$ ). Elle emploie entre 6 à 7 pêcheurs.

- Apollo motorisé
Depuis le début des années 90, une forme avancée d'unités catamaran a fait son apparition au Burundi, unité Apollo. Cette unité se compose de trois pirogues en bois de $9 \mathrm{~m}$ de long composé des perches de $12 \mathrm{~m}$ environ, équipée autrefois d'une dizaine de lampes (actuellement 14) et propulsée par un moteur de 25 à $50 \mathrm{CV}$. Le filet carrelet utilisé pour les unités Appolo permet quant à lui de développer une pêche beaucoup plus importante avec une circonférence de $110 \mathrm{~m}$ pour une chute de $25 \mathrm{~m}$. Une unité Appolo coûte environ 5.200\$ US (ou $9880000 \mathrm{FBu}$ ) et emploie 8 à 9 personnes.

Les résultats de la figure 10 montrent que quatre types de moteurs de 9,9 à $40 \mathrm{CV}$ sont utilisés à Mvugo et seulement trois types à Muguruka. La plage de Mvugo dispose de plus de moteurs HP $40 \mathrm{CV}$ tandis qu'à Muguruka, ce sont les moteurs HP 15 CV qui dominent.

Toutes les unités de pêche artisanale qui débarquent à Muguruka et à Mvugo utilisent des lampes pour attirer les poissons en général et les clupéidés en particulier qui, à leur tour attirent leurs prédateurs. Le nombre moyen de lampes de pêche a été respectivement évalué à $12 \pm 4$ pour le catamaran non motorisé, de $14 \pm 4$ et $16 \pm 4$ pour l'unité Appolo sans différence dans leur durée d'utilisation (2.4 \pm 0.8 ans) par lampe. Le Tableau 6 montre que le nombre total de lampes inventoriées à Muguruka à augmenter pour tenter d'augmenter les captures par unité d'effort. A Mvugo, par contre, les captures globales diminuant, le nombre d'unités de pêche diminue, ce qui se traduit donc par une diminution du total des lampes inventoriées.

Les résultats du Tableau 7 montrent qu'une unité catamaran non motorisée nécessite un investissement d'au moins 3.640 \$US alors qu'une unité catamaran motorisée fonctionne avec un investissement de 6.160 \$US dans le cas où elle utilise un moteur HP de $15 \mathrm{CV}$. Quant à l'unité Appolo ou trimaran motorisé, le coût d'investissement s'élève à environ 11.430 \$US. 
Les coûts d'exploitation des unités de pêche artisanales à Mvugo comme à Muguruka s'élèvent à \pm 3.100 \$US pour le catamaran non motorisé, il est d'au moins 17.500 \$US pour le catamaran motorisé alors que pour l'unité Appolo motorisée, le C.E. s'élève à environ 22.960 \$US).

La CPUE moyenne de l'unité Appolo s'élève à $152 \mathrm{~kg} /$ nuit, à $80 \mathrm{~kg} /$ nuit pour le catamaran motorisé et à $50 \mathrm{~kg} /$ nuit pour le catamaran non motorisé. Après la vente de ces produits de pêche, le profit net annuel estimé par unité de pêche artisanale s'élève à $8.300 \pm$ 2.230 \$US avec un revenu net par pêcheur d'environ 691 \$US pour le catamaran non motorisé, il est de $4.380 \pm 650$ \$US pour le catamaran motorisé, avec un revenu net par pêcheur de (soit environ 275 \$US, tandis que pour l'unité Appolo, le P.N.E. s'élève à $11.670 \pm 2.500$ \$US avec un C.S par pêcheur de $645 \pm 140$ \$US.

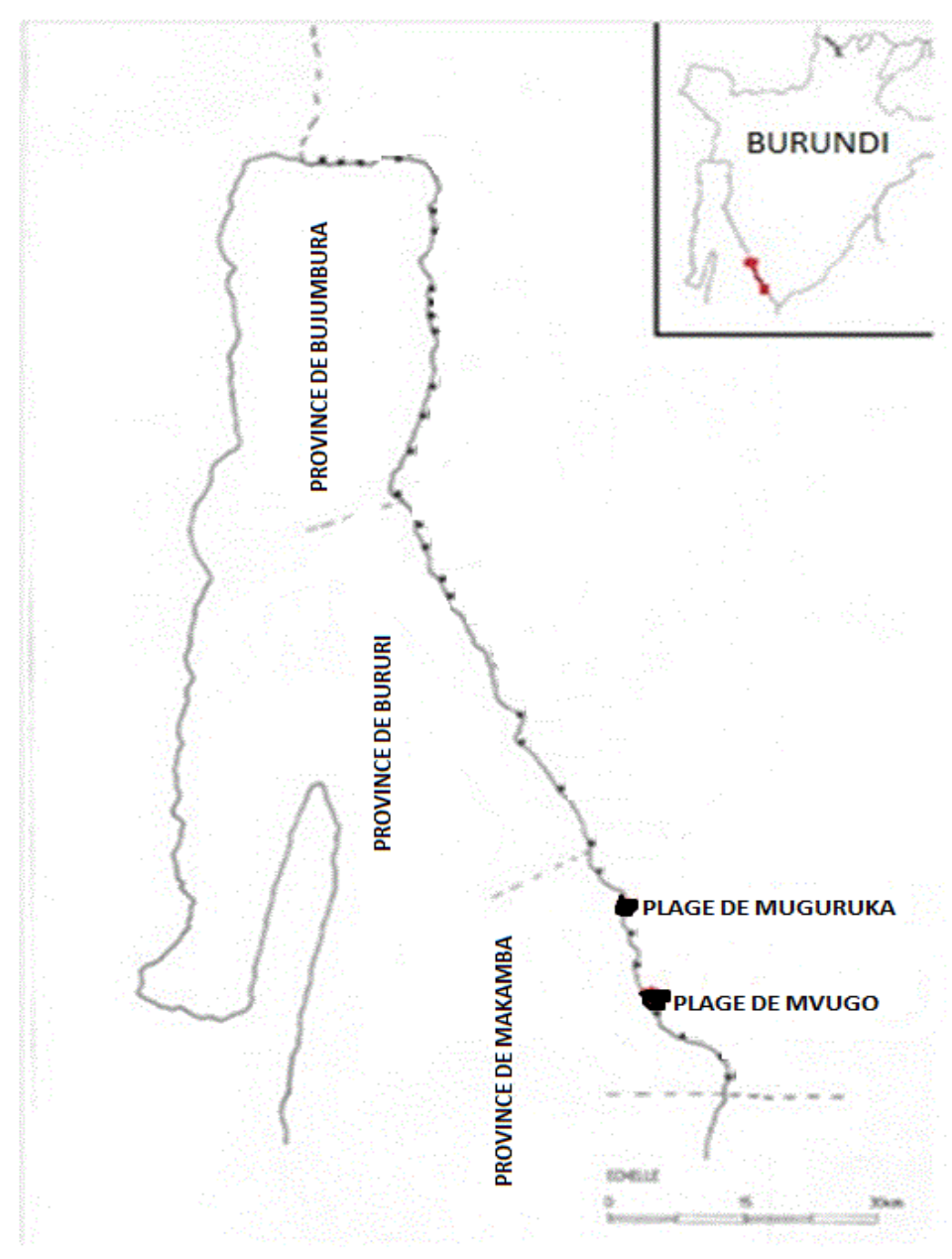

Figure 1 : Sites d'échantillonnage (Muguruka et Mvugo) des poissons dans les eaux burundaises au Nord-Est du lac Tanganyika. 
Tableau 1: Variations du nombre d'unités de pêche artisanale actives débarquant à Muguruka pendant la période de mars à juillet 2013 par rapport aux inventaires de 2011 (PRODAP, 2011) et aux données de 2012 du Département des Eaux, Pêche et Pisciculture.

\begin{tabular}{llll}
\hline \multicolumn{4}{l}{ Muguruka } \\
\hline UPA & PRODAP 2011 & DPPB 2012 & NE 2013 \\
\hline Cat. non mot. & $\mathbf{1 8}$ & $\mathbf{2 7}$ & $\mathbf{3 2}$ \\
variation en \% & 100 & 150 & 177,8 \\
Evolution en \% & 0 & $(+50 \%)$ & $(+77,8 \%)$ \\
Cat. mot. & $\mathbf{1 1}$ & 7 & 7 \\
Variation en \% & 100 & 63,6 & 63,6 \\
Evolution en \% & 0 & $(-36,4 \%)$ & $(-36,4 \%)$ \\
Appolo & $\mathbf{2 3}$ & $\mathbf{2 0}$ & $\mathbf{1 5}$ \\
Variation en \% & 100 & 86,9 & 65,2 \\
Evolution en \% & 0 & $(-13,1 \%)$ & $(-34,8 \%)$ \\
\hline UPA = Unité de pêche artisanale, DEPP = Département des Eaux, Pêche et Pisciculture du Burundi, Cat. non mot. $=$ \\
Catamaran non motorisé, Cat. mot. $=$ catamaran motorisé, NE 2013 = Note enquête de mars à juillet 2013, - : réduction et + : \\
augmentation.
\end{tabular}

Tableau 2: Variations du nombre d'unités de pêche artisanale actives débarquant à Mvugo pendant la période de mars à juillet 2013 par rapport aux inventaires de 2011 (PRODAP, 2011) et aux données de 2012 du Département de Pêche et Pisciculture du Burundi (DEPP).

\begin{tabular}{llll}
\hline & Mvugo & & \\
\hline UPA & PRODAP 2011 & DPPB 2012 & NE 2013 \\
\hline Cat. non mot. & $\mathbf{3 7}$ & $\mathbf{4 8}$ & $\mathbf{6 0}$ \\
variation en \% & 100 & 129,7 & 162,2 \\
Evolution en \% & 0 & $(+29,7)$ & $(+62,2)$ \\
Cat. mot. & $\mathbf{3}$ & $\mathbf{5}$ & $\mathbf{7}$ \\
Variation en \% & 100 & 166,7 & 233,3 \\
Evolution en \% & 0 & $(+66,7)$ & $(+133,3)$ \\
Appolo & $\mathbf{1 1 1}$ & $\mathbf{7 0}$ & $\mathbf{5 0}$ \\
Variation en \% & 100 & 63,1 & 45,1 \\
Evolution en \% & 0 & $(-36,9)$ & $(-54,9)$ \\
\hline
\end{tabular}

Tableau 3 : Mailles et types de filets utilisés à Muguruka et Mvugo, lac Tanganyika, Burundi.

\begin{tabular}{llllll}
\hline Mailles & Car. & F.M & S.P. & Total & \% \\
\hline$<4 \mathrm{~mm}$ & 100 & 19 & 97 & 216 & 45,2 \\
4 à $5 \mathrm{~mm}$ & 44 & 35 & 65 & 144 & 30,1 \\
\multicolumn{1}{c}{$>\quad 5 \mathrm{~mm}$} & 27 & 48 & 43 & 118 & 24,7 \\
\hline Total & 171 & 102 & 205 & 478 & 100,0 \\
\hline$\%$ & 35,8 & 21,3 & 42,9 & 100,0 & \\
\hline
\end{tabular}

Car $=$ Carrelet, $\mathrm{F} . \mathrm{M}=$ Filet Maillant, $\mathrm{SP}=$ Senne de Plage 


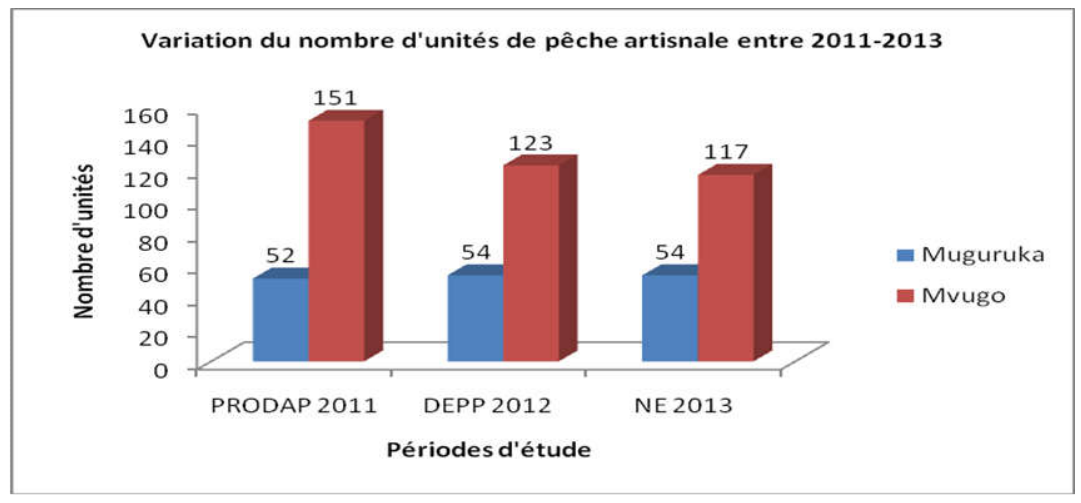

Figure 2:Variation du nombre d'unités de pêche artisanale de 2011 à 2013 à Muguruka et à Mvugo, N-E lac Tanganyika, Burundi.

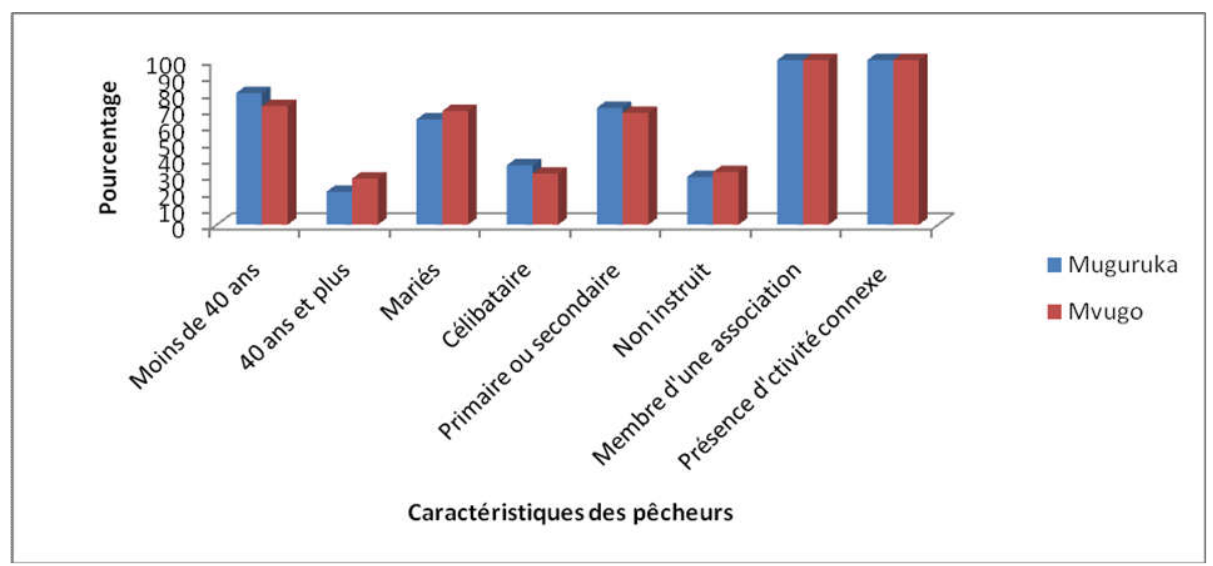

Figure 3 : Inventaire, situation et caractéristique des pêcheurs par site de débarquement au lac Tanganyika (plages de Muguruka et Mvugo, Burundi).

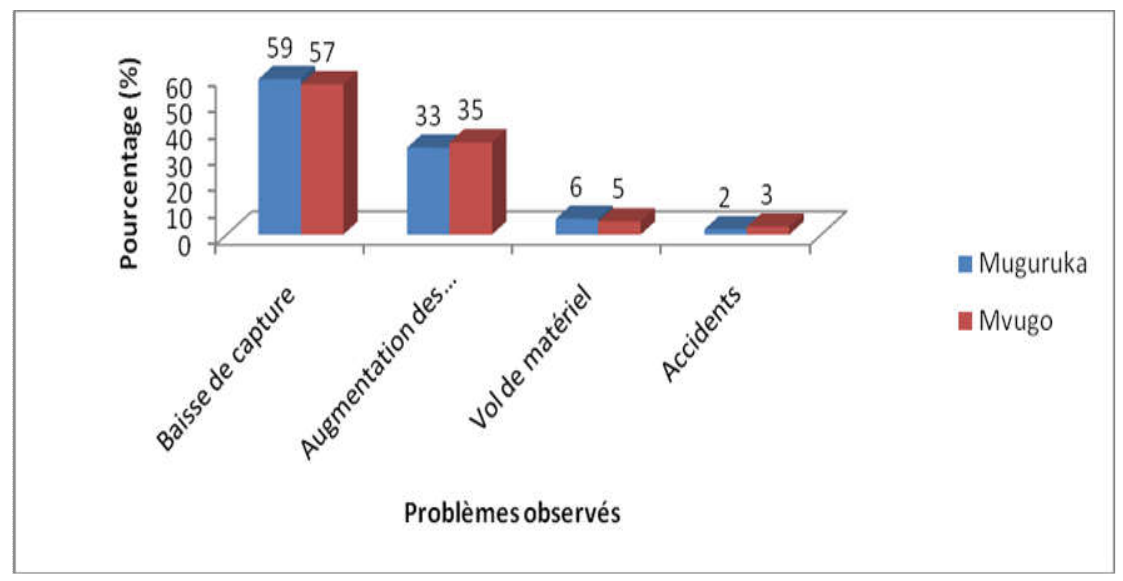

Figure 4: Problèmes soulevés par les pêcheurs dans les deux sites de débarquement au Lac Tanganyika (plages de Muguruka et Mvugo, Burundi). 


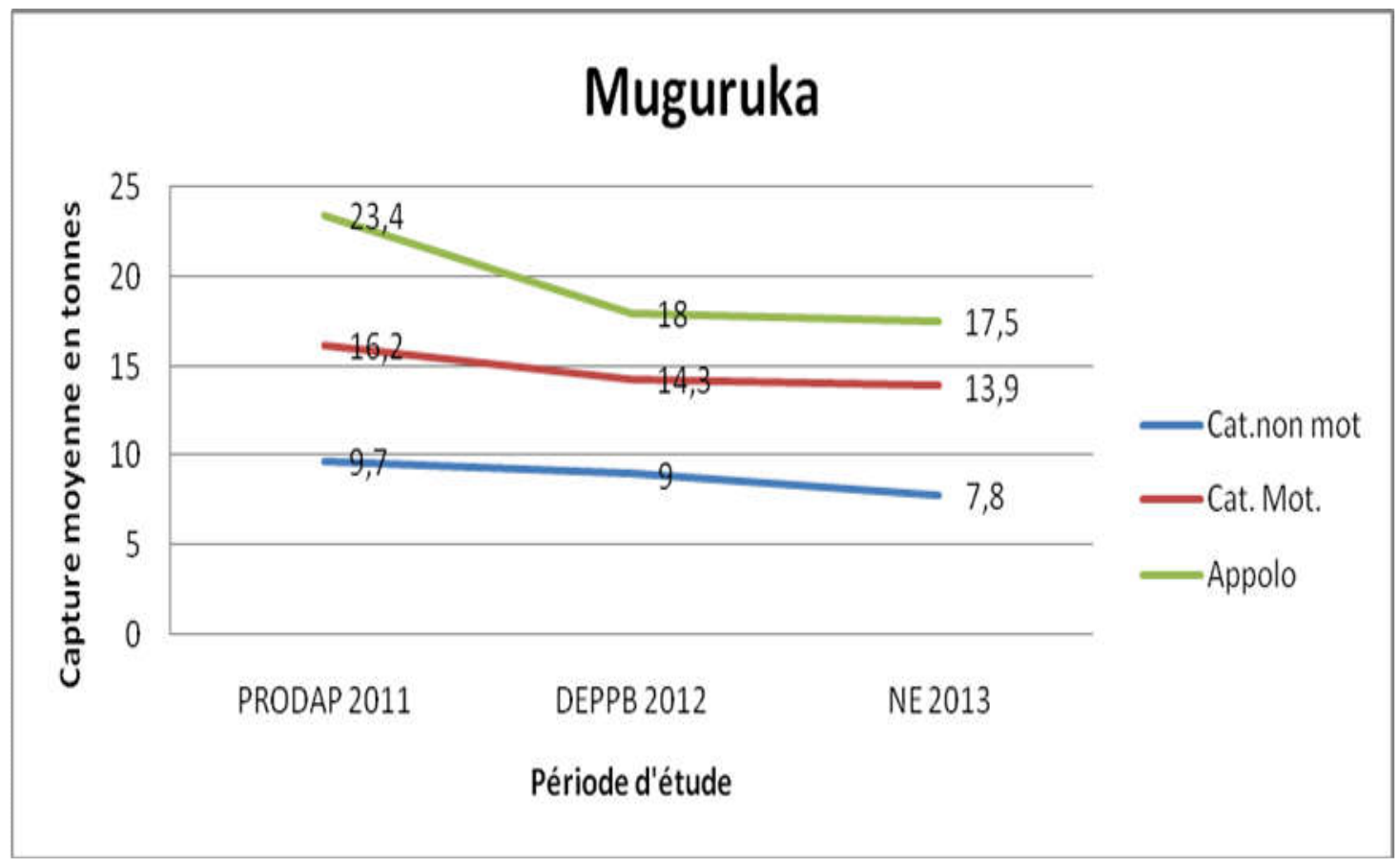

Figure 5 : Evolution de la capture moyenne annuelle (en tonnes) de 2011 à 2013 par les différentes unités de pêche artisanale au site de débarquement de Muguruka, Lac Tanganyika, Burundi.

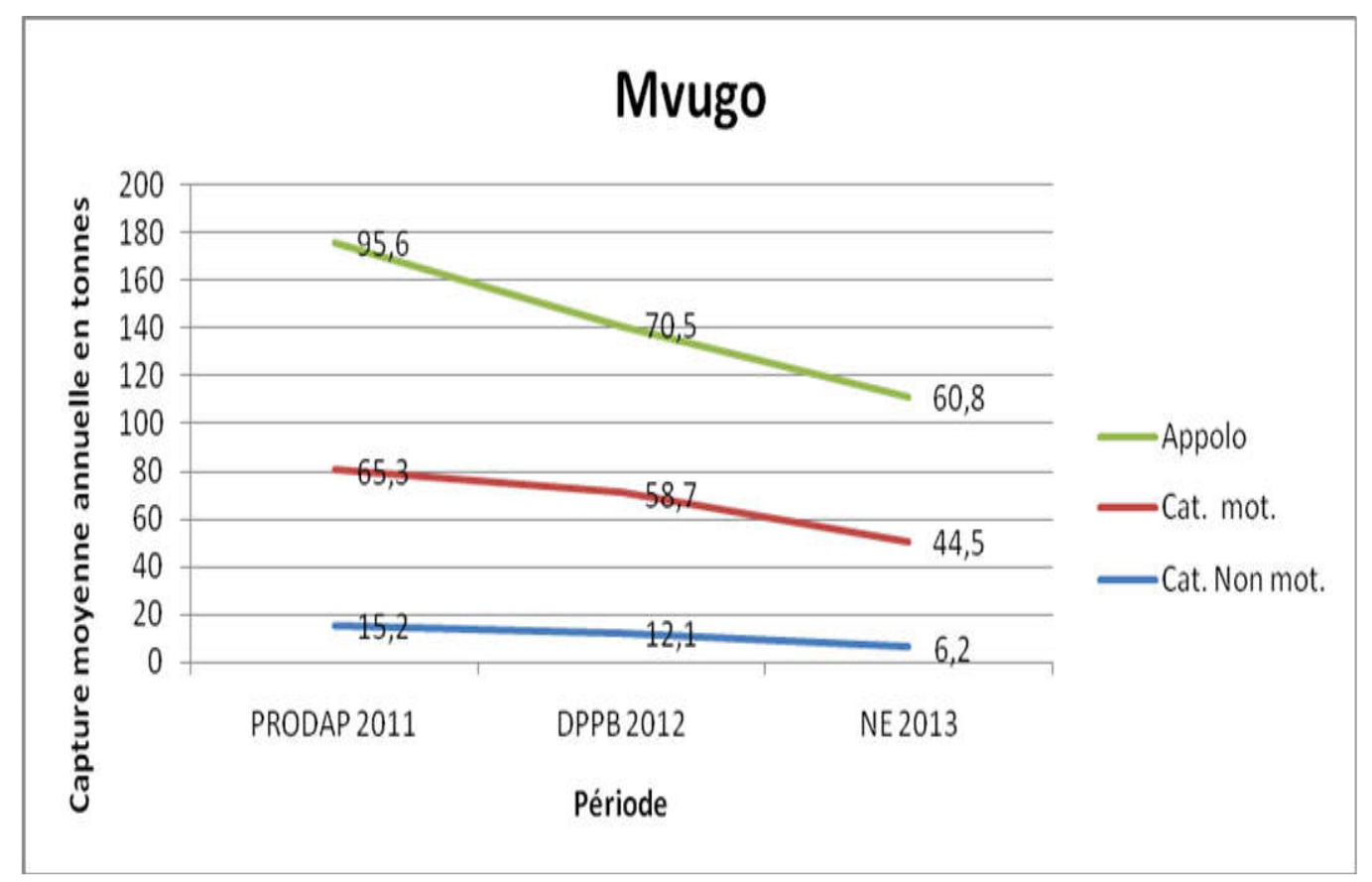

Figure 6 : Evolution de la capture moyenne annuelle (en tonnes) de 2011 à 2013 par les différentes unités de pêche artisanale au site de débarquement de Mvugo, Lac Tanganyika, Burundi. 
Tableau 4 : Corrélations entre le nombre d'unités de pêche artisanale, de lampes utilisées et les Captures Par Unité d'Effort de 2011 à 2013.

\begin{tabular}{|c|c|c|c|c|c|c|c|c|c|}
\hline VARIABLES & UPA2011 & CPUE2011 & LAMPE2011 & UPA2012 & CPUE2012 & LAMPE2012 & UPA2013 & CPUE2013 & LAMPE2013 \\
\hline UPA2011 & 1 & & & & & & & & \\
\hline CPUE2011 & 0,673 & 1 & & & & & & & \\
\hline LAMPE2011 & 0,999 & 0,658 & 1 & & & & & & \\
\hline UPA2012 & $\mathbf{0 , 9 2 2}$ & 0,442 & 0,919 & 1 & & & & & \\
\hline CPUE2012 & 0,566 & 0,9 & 0,547 & 0,332 & 1 & & & & \\
\hline LAMPE2012 & 0,912 & 0,404 & 0,913 & 0,997 & 0,291 & 1 & & & \\
\hline UPA2013 & 0,67 & 0,126 & 0,663 & 0,902 & 0,38 & 0,9 & 1 & & \\
\hline CPUE2013 & 0,614 & $\mathbf{0 , 9 3 3}$ & 0,6 & 0,351 & 0,99 & 0,316 & 0,15 & 1 & \\
\hline LAMPE2013 & 0,647 & 0,74 & 0,642 & 0,89 & $-0,16$ & 0,895 & 0,996 & $-0,35$ & 1 \\
\hline
\end{tabular}

(UPA=Unité de pêche artisanale, CPUE = Capture Par Unité d'Effort, LAMPE = Nombre de lampes utilisées. Les chiffres en gras sont les corrélations significatives à $\mathrm{p}>0,01$ tandis que les chiffres en italique et en gras sont des corrélations significatives à $\mathrm{p}>0,05$.

Tableau 4 : Fréquence de tailles $(\mathrm{mm})$ des poissons capturés par espèce aux sites de débarquement de Muguruka et Mvugo, lac Tanganyika, Burundi en 2013.

\begin{tabular}{lllll}
\hline \multirow{2}{*}{ Espèces } & Nombre & \multicolumn{3}{l}{ Taille moyenne (mm) } \\
\cline { 2 - 5 } & Muguruka & Mvugo & Muguruka & Mvugo \\
\hline Limnothrissamiodon & 1054 & 1096 & $79 \pm 23$ & $78 \pm 20$ \\
Latesstappersii & 571 & 1194 & $121 \pm 101$ & $123 \pm 99$ \\
Stolothrissatanganicae & 1225 & 1260 & $64 \pm 18$ & $67 \pm 15$ \\
\hline Total & $\mathbf{2 8 5 0}$ & $\mathbf{3 5 5 0}$ & & \\
\hline
\end{tabular}

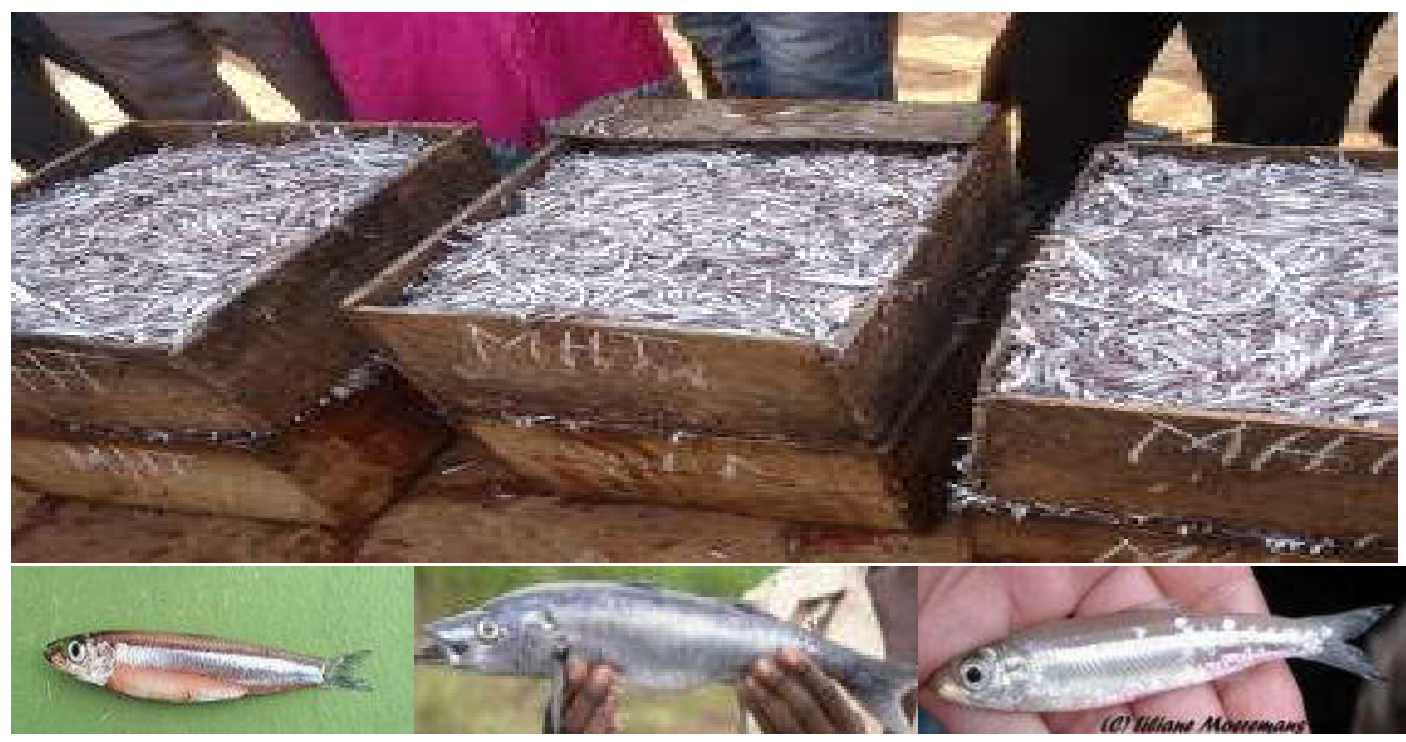

Photo 1: Caisses pleines de sardines (haut) et de gauche à droite (bas) : Limnothrissa miodon, Luciolates stappersii et Stolothrissa tanganicae. 

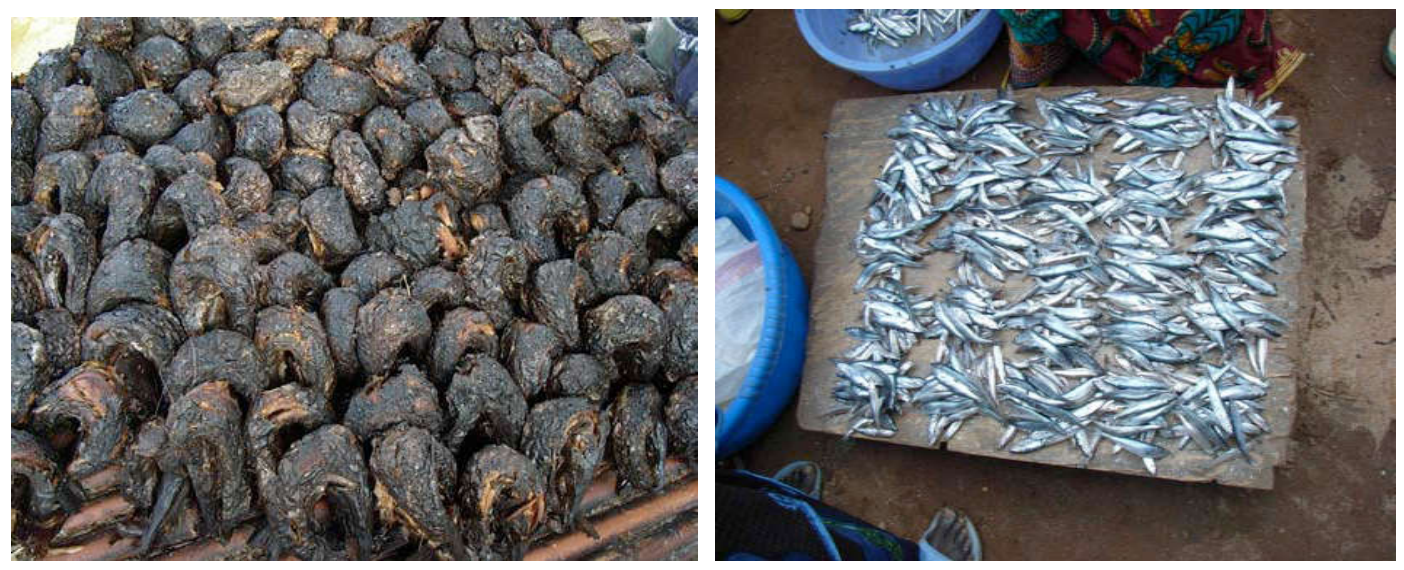

Photo 2: Lates stappersii fumés (à gauche) et étalage des clupéidés (à droite) au marché de Bujumbura.

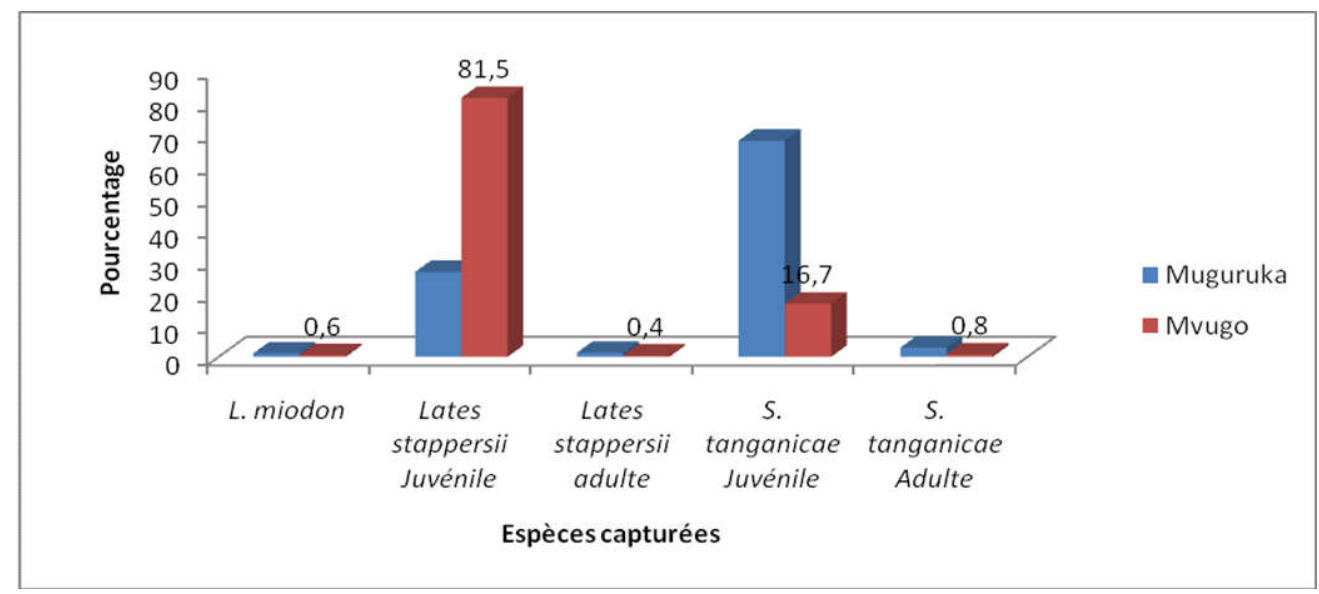

Figure 7: Pourcentage des espèces de poissons capturées aux plages de débarquement de Muguruka et Mvugo, Lac Tanganyika, Burundi en 2013.

Tableau 5 : Comparaison des taux de captures des différentes taxa par maille de filets utilisés.

\begin{tabular}{llllll}
\hline Variables & Maille1 & Maille2 & Maille3 & Total & \% \\
\hline Taxon 1 & 2165 & 1665 & 80 & $\mathbf{3 9 1 0}$ & 9,2 \\
Taxon2 & 4905 & 3769 & 1786 & $\mathbf{1 0 4 6 0}$ & 24,5 \\
Taxon3 & 230 & 180 & 90 & $\mathbf{5 0 0}$ & 1,2 \\
Taxon4 & 200 & 405 & 515 & $\mathbf{1 1 2 0}$ & 2,6 \\
Taxon5 & 11990 & 9330 & 5329 & $\mathbf{2 6 6 4 9}$ & 62,5 \\
\hline Total & $\mathbf{1 9 4 9 0}$ & $\mathbf{1 5 3 4 9}$ & $\mathbf{7 8 0 0}$ & $\mathbf{4 2 6 3 9}$ & 100,0 \\
\hline$\%$ & 45,7 & 36 & 18,3 & $\mathbf{1 0 0}$ & \\
\hline
\end{tabular}

Maille1 < $4 \mathrm{~mm}$, Maille2 $=4$ à $5 \mathrm{~mm}$, Maille3 $>5 \mathrm{~mm}$; Taxon 1: Limnothrissa miodon, Taxon 2: Juvéniles de Lates stappersii, Taxon 3: Lates stappersii, Taxon 4: Stolothrissa tanganicae (adultes), Taxon 5: Stolothrissa tanganicae (juvéniles). 
Tableau 6 : Nombre de lampes utilisées par les unités de pêche artisanale (UPA) aux deux sites de débarquement au Lac Tanganyika (plages du sud Burundi) moyenne et écart-type durant la période de 2011 à 2013.

\begin{tabular}{llll}
\hline Muguruka & & & \\
\hline UPA & PRODAP 2011 & DPPB 2012 & NE 2013 \\
\hline Cat. Non mot. & $216 \pm 72$ & $324 \pm 108$ & $384 \pm 62$ \\
Cat. mot. & $154 \pm 44$ & $98 \pm 28$ & $98 \pm 28$ \\
Appolo & $368 \pm 92$ & $320 \pm 80$ & $240 \pm 60$ \\
\hline Total & $\mathbf{7 3 8} \pm \mathbf{2 0 8}$ & $\mathbf{7 4 2} \pm \mathbf{2 1 6}$ & $\mathbf{7 2 2} \pm \mathbf{1 5 0}$ \\
\hline & Mvugo & & \\
\hline UPA & PRODAP 2011 & DPPB 2012 & NE 2013 \\
\hline Cat. Non mot. & $444 \pm 148$ & $576 \pm 192$ & $720 \pm 240$ \\
Cat. mot. & $42 \pm 12$ & $70 \pm 20$ & $98 \pm 28$ \\
Appolo & $1776 \pm 444$ & $1120 \pm 280$ & $800 \pm 200$ \\
\hline & & & $\mathbf{1 6 1 8}$ \\
Total & $\mathbf{2 2 6 2} \pm \mathbf{6 0 4}$ & $\mathbf{1 7 6 6 \pm \mathbf { 4 9 2 }}$ & $\mathbf{4 6 8}$ \\
\hline
\end{tabular}

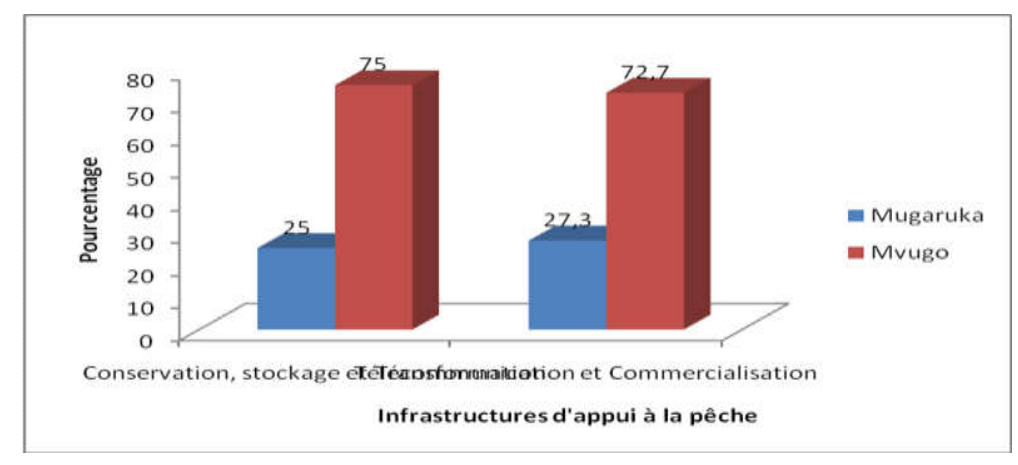

Figure 8 : Infrastructures d'appui à la pêche dans les deux sites de débarquement Muguruka et Mvugo, lac Tanganyika, Burundi.

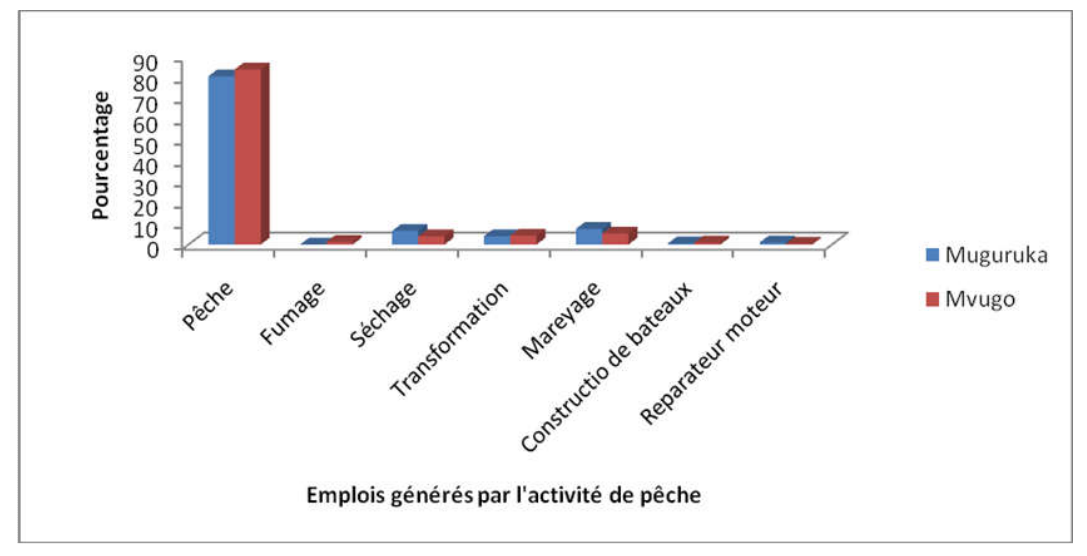

Figure 9 : Emplois générés par les activités de pêche aux sites de débarquement de Muguruka et de Mvugo, lac Tanganyika, Burundi. 


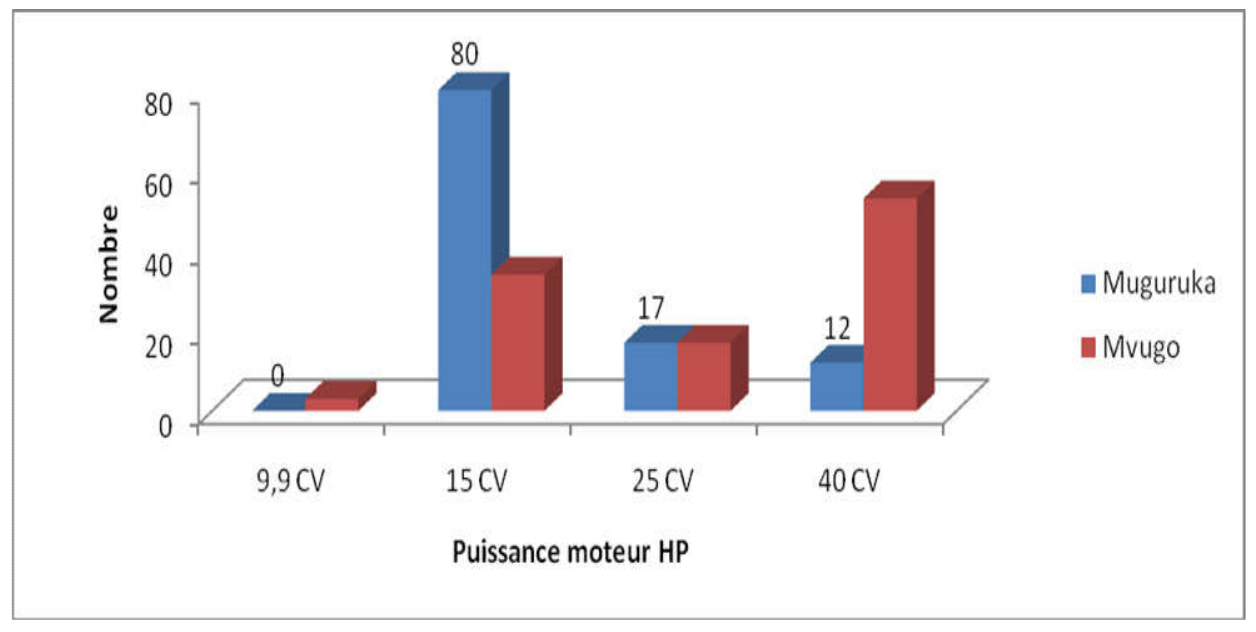

Figure 10 : Puissance des moteurs utilisés par site de débarquement au Lac Tanganyika (plages du sud Burundi).

Tableau 7 : Indices de performance économique (en \$US) des unités de pêche artisanale de la côte sud burundaise du Lac Tanganyika en 2013.

\begin{tabular}{|c|c|c|c|c|}
\hline I.P.E. & C.I. (\$US) & C.O. (\$US) & Am. (\$US) & C.E. (\$US) \\
\hline Cat. Non Mot. & $3.640 \pm 760$ & $3.650 \pm 980$ & $550 \pm 100$ & $3.100 \pm 880$ \\
\hline Cat. Mot. & $6.160 \pm 790$ & $18.550 \pm 5.440$ & $1.050 \pm 150$ & $17.500 \pm 5.300$ \\
\hline Appolo & $11.430 \pm 1.990$ & $24.910 \pm 7.470$ & $1.940 \pm 380$ & $22.960 \pm 7.090$ \\
\hline
\end{tabular}

I.P.E. = Indices de Performance Economique, Cat. Non Mot.= Catamaran non motorisé, Cat. Mot.= Catamaran motorisé, C.I.=Coût d'investissement : valeur actuelle de toute l'unité de pêche artisanale, C.O. = Coût d'opportunité : revenu que le propriétaire peut obtenir du capital investi dans la pêche, Am. $=$ Amortissement, C.E. $=$ Coût d'exploitation : coût d'opportunité moins l'amortissement.

Tableau 7 : Capture annuelle Par Unité d'Effort de Pêche (CPUE) et rentabilité économique (en \$US) des différentes unités de pêche artisanale du lac Tanganyika (plages du sud burundais).

\begin{tabular}{llllllll}
\hline UPA & CPUE (kg) & P. (N/m) & P.B.E. & P.N.E. & N.P. & C.S. & T.R. \\
\hline Cat. non mot. & $50 \pm 15$ & $12 \pm 1$ & $11.400 \pm 3.100$ & $8.300 \pm 2.230$ & $6 \pm 2$ & $691 \pm 185$ & $228 \%$ \\
Cat. Mot. & $80 \pm 23$ & $10 \pm 1$ & $21.800 \pm 5.950$ & $4.380 \pm 650$ & $8 \pm 2$ & $275 \pm 40$ & $71 \%$ \\
Appolo & $152 \pm 44$ & $10 \pm 1$ & $34.640 \pm 9.600$ & $11.670 \pm 2.500$ & $9 \pm 2$ & $645 \pm 140$ & $102 \%$ \\
\hline
\end{tabular}

Moyenne \pm écart-type $; \mathrm{UPA}=$ Unités de pêche artisanale, $\mathrm{P}(\mathrm{N} / \mathrm{m})=$ Pêche (Nuits/mois), P.B.E. $=$ Profit Brut Estimé, P.N.E.

$=$ Profit Net Estimé, N.P= Nombre de pêcheurs, C.S. $=$ Coût Salarial, T.R. $=$ Taux de Rentabilité. 


\section{DISCUSSION \\ Caractérisation des pêcheurs et des unités de pêche}

Nos résultats montrent que la pêche artisanale au Lac Tanganyika joue des rôles importants au Burundi en termes d'emploi, de sécurité alimentaire, d'avantages sociaux et économiques ce qui confirme les résultats obtenus à Uvira et Fizi en RD Congo par Mushagalusa et al. (2015), qui sont des centres majeurs de pêche au nord-ouest du Lac Tanganyika (FAO, 2009 ; Lindley, 2000). Le secteur de la pêche artisanale emploie donc beaucoup de personnes dont plus de $80 \%$ sont des pêcheurs, les autres $(20 \%)$ se répartissent dans les activités de stockage, de conservation (séchage solaire), de transformation et de commercialisation.

Quand on compare les données collectées pendant la dernière enquête cadre de la pêche au Lac Tanganyika (Prodap, 2011), le nombre de pêcheurs et d'unités de pêche artisanale débarquant a fortement baissé de $44,9 \%$ et $35 \%$ pour les catamarans respectivement à Muguruka et à Mvugo. Quant aux unités Appolo, elles ont aussi fortement baissé de $65,3 \%$ à Muguruka et de $48,7 \%$ à Mvugo.

Ceci résulterait du fait que les dépenses investies dans la pêche par la plupart d'unités motorisées (Catamaran et Appolo) ne sont pas récupérées ; alors que les coûts de production, dominés par celui du carburant pour les moteurs hors-bords et le pétrole pour les lampes, ont considérablement augmenté, la capture ne fait que diminuer et le prix du poisson est resté stable $(3.200 \mathrm{FBu} / \mathrm{kg}$ de $L$. miodon, $5.500 \mathrm{FBu} / \mathrm{kg}$ de $S$. tanganicae et $7.000 \mathrm{FBu} / \mathrm{kg}$ de L. stappersii en 2011 comme en 2013), limité par le faible pouvoir d'achat des consommateurs. Le même constat a été fait aussi du côté congolais du Lac Tanganyika à Uvira et Fizi (Mushagalusa et al., 2014).

\section{Effort de pêche et commerce}

En ce qui concerne le coût d'une unité de pêche artisanale, le moteur (32 à 38\%) représente la plus grande partie de l'investissement (Yamaha $40 \mathrm{CV}: 4.325$ \$US, Mariner 25 CV : 3.460 \$US, Yamaha 15 CV: 2.030 \$US). Les pirogues en planches pour le catamaran $(32 \%)$ et le trimaran ou Appolo $(43 \%)$ et le filet carrelet $(9,4 \%)$ viennent respectivement en deuxième et troisième position. Les autres matériels complètent ces gros constituants. Il s'agit des lampes, cordages, toile imperméable, perches, caisse en planches et porte-lampes.

Les résultats précédents de Breuil (1995) indiquaient que le coût moyen d'un catamaran motorisé était de 3600 \$US et de 5200 \$US pour le trimaran ou Appolo au Burundi. Pour qu'une unité catamaran non motorisé fonctionne actuellement au Burundi, il faut investir de l'ordre de 3.640 \$US alors qu'une unité catamaran motorisé fonctionne après un investissement de 6.160 \$US dans le cas où elle utilise un petit moteur HP de 15 $\mathrm{CV}$. Quant à l'unité Appolo ou trimaran, le coût d'investissement s'élève à 11.430 \$US. On constate donc sur une vingtaine d'années, un fort accroissementdes coûts d'investissement pour cette pêche artisanale des petits poissons pélagiques. Quant aux coûts d'exploitation des unités de pêche artisanale (UPAs) à Mvugo comme à Muguruka, ils s'élèvent à environ 3.100\$US pour le catamaran non motorisé, 17.500 \$US pour le catamaran motorisé alors que pour l'unité Appolo, le C.E. s'élève 22.960 \$US. Ces résultats vont dans le même sens et corroborent ceux de Mushagalusa et al. (2015) à Uvira et Fizi en République Démocratique du Congo où l'investissement total pour chaque unité de pêche artisanale est de 5.112 \$ US et 6740 \$US respectivement pour l'unité Catamaran et Appolo. Toutefois, malgré un investissement plus élevé et des charges d'exploitation plus importantes, l'unité Appolo aux plages burundaises de Muguruka et Mvugo, obtient un chiffre d'affaires mensuel légèrement plus haut (2.556 US\$) que le catamaran motorisé (2.496 US\$) et le non motorisé (1.950 US\$). En termes de revenu annuel par pêcheur, nos résultats 
montrent que le meilleur revenu était assuré par les pêcheurs de catamarans non motorisés.

Des études précédentes ont stipulé que le catamaran et Appolo étaient équipés de filets de 70 et $110 \mathrm{~m}$ et de 6 à 8 ou 10 lampes (Mannini et al., 1997 ; Herve et Dominique, 2010 ; Mushagalusa et al., 2015). Ces tailles de filet sont restées approximativement les mêmes qu'actuellement tandis que le nombre de lampes par unité artisanale de pêche a remarquablement doublé ces deux dernières décennies $(12 \pm 4$ pour le catamaran non motorisé, $14 \pm 4$ pour le catamaran motorisé et $16 \pm 4$ pour l'unité Appolo et cela dans les deux sites, à Mvugo comme à Muguruka). Par conséquent, la consommation de pétrole des lampes pour les unités de pêche artisanale a augmenté proportionnellement à leur nombre. Le pétrole des lampes et le carburant pour les moteurs des unités Appolo et Catamaran motorisé, en plus des investissements initiaux plus conséquents, ont rendus ces unités plus chères (augmentation des coûts fixes comme des coûts variables) que le catamaran non motorisé et donc moins rentable.

Les prises de poisson du Lac Tanganyika sont principalement reflétées par les changements de pratiques de pêche ou d'effort de pêche. Ainsi, depuis les années 1990, l'observation la plus commune est la diminution des CPUE observée pour toutes les unités de pêche artisanale (Sarvalaet al., 2006; Van der Knaap et al., 2014). C'est ce que nous observons aussi dans la partie nord du lac, aussi bien du côté ouest congolais à Uvira et Fizi (Mushagaluza et al., 2015) que du côté burundais à Mvugo et Muguruka. Ainsi, le nombre de catamarans non motorisés, motorisés et de trimaran Appolo a baissé par rapport à l'enquête cadre de 2011, respectivement de $59 \%, 20 \%$ et $64 \%$.

Les CPUE moyennes de l'unité Appolo s'élève à $152 \mathrm{~kg} /$ nuit, elle est de $80 \mathrm{~kg} /$ nuit pour le catamaran motorisé et de $50 \mathrm{~kg} /$ nuit pour le catamaran non motorisé. Après la vente de ces produits de pêche, le profit annuel net estimé par unité de pêche artisanale s'élève à 8.300\$US avec un revenu annuel net par pêcheur de 691 \$US pour le catamaran non motorisé, il est de 4.400 \$US, avec un revenu annuel net par pêcheur d'au moins 275 \$US, tandis que pour l'unité Appolo, le profit net estimé par an s'élève à 11.670 \$US avec un coût salarial annuel par pêcheur d'au moins 645 \$US. Les résultats de Mushagalusa et al. (2014, 2015) montrent que le catamaran non motorisé est le bateau le plus commun à Uvira et Fizi en République Démocratique du Congo avec une capture moyenne de $135 \mathrm{~kg}$ /nuit et un revenu net de 901 \$US par pêcheur contre une capture moyenne de $124 \mathrm{~kg} /$ nuit et un revenu de 445,5 \$US par pêcheur pour le trimaran ou Appolo. Ces fluctuations peuvent résulter de variations dans le recrutement naturel des espèces, dans leurs déplacements mais aussi et surtout de leur surexploitation car le phénomène s'observe partout (nordouest et nord-est du lac, comme rapporté aussi par Sarvala et al. (2006).

Il en résulte que, bien que les augmentations des coûts de la pêche artisanale des petits pélagiques s'observent au Burundi comme au Congo (nord du Lac Tanganyika), la rentabilité de la pêche est meilleure en catamaran non motorisé dans la partie nord du Lac Tanganyika alors que les plus grosses unités à plus gros moteurs (Appolo) coûtent cher dans les deux pays. Ceci résultede la hausse d'intrant de la pêche d'une part, et de la baisse des captures actuellement observée sur l'ensemble du lac. Les pêcheurs commencent à bien le comprendre en réduisant fortement leur nombre annuel de sorties de pêche, en réduisant le nombre des unités de pêche et en cherchant à survivre dans d'autres activités que la pêche. Il serait temps pour assurer la durabilité de la pêche au Lac Tanganyika de limiter l'accès à la ressource en délivrant enfin un nombre limité de permis de pêche adapté à ce que la ressource peut supporter.

\section{Conclusion}

La synthèse des informations obtenues pour cette étude, montre que :

- La pêche est une activité qui contribue à l'amélioration des conditions de vie de la population du littoral du Lac Tanganyika. Elle 
a contribué à la création d'emploi de pêcheurs $(80 \%)$, de mareyeurs, de transformateurs et de commerçants et au développement d'assez bonnes infrastructures (chambres froides, aire de séchage solaire sur grillage, fumoir performant, petit marché), du moins aux plages de Muguruka et Mvugo ;

- Plus il y a d'unités, plus il y a de nombre de lampes à utiliser sans qu'il y ait une augmentation de la capture ;

- Partout au Lac Tanganyika, les Captures Par unité d'Effort de Pêche (CPUE) diminuent, le nombre d'unités actives de pêche se réduit par manque de poissons, la rentabilité des unités de pêche se réduit, les revenus des pêcheurs diminuent.

- Les pêcheurs sont organisés sur chaque plage avec un comité de plage qui défend leursintérêts dans la mesure du possible et qui organise et limite les sorties pour réduire la pression sur les stocks ;

- Les poissons sont commercialisés selon un circuit simple où tous les pêcheurs sont regroupés au sein d'une association (AFPOMABU) qui assure la distribution et optimise la vente des poissons vers les grands centres.

Il serait important que l'administration burundaise des pêches mette en place à l'avenir :

$$
\begin{gathered}
\checkmark \quad \text { des structures adéquates de } \\
\text { financement des pêcheurs; } \\
\checkmark \quad \text { des infrastructures adéquates dans } \\
\text { toutes les plages de débarquement; } \\
\checkmark \quad \text { un dispositif de contrôle de la qualité } \\
\text { des poissons avant et après } \\
\quad \text { transformation; } \\
\checkmark \quad \text { la participation des communautés } \\
\text { locales et organisations socio professionnelles } \\
\text { de pêcheur dans la conception, l'exécution, le } \\
\text { contrôle et le suivi de leurs activités de pêche. }
\end{gathered}
$$

\section{REMERCIEMENTS}

Nous remercions le personnel de l'Institut Supérieur Agrovétérinaire et de Conservation de la nature, (ISAVC/Minembwe) pour son support administratif. Nos remerciements vont aussi, d'une part, aux enquêteurs de pêche sur le terrain et aux responsables du DEPP du Burundi pour les données fournies, et d'autre part, aux pêcheurs et à leurs présidents d'association qui ont accepté de collaborer et de répondre à notre questionnaire.

\section{RÉFÉRENCES}

Allgayer R. 1992. Les eaux bleues du Lac Tanganyika. La Revue Française d'Aquariologie, Paris/France : 9-11.

Bongeba C, Micha JC. 2013. Etat de la pêche au sud du lac Maï-Ndombe, Revue Scientifique et Technique Forêt et Environnement du Bassin du Congo, 1 : 46-55.

Breuil C. 1995.Pêcheries de pélagiques du Lac Tanganyika : étude des aspects économiques. FAO Circulaire sur les pêches $\mathrm{N}^{\circ}$ 899. FIPP/C899, Rome, 1995, $48 \mathrm{p}$.

Coenen EJ, Paffen P, NIKOMEZE, 1998. Catch per unit of effort (CPUE) study for different areas fishing gears of Lake Tanganyika. FAO/FINNIDA Research for Management of Fisheries on Lake Tanganyika. GCP/RAF/271/FIN-TD/80, $86 \mathrm{p}$.

Collart A 1985. Pêche artisanale et pêche industrielle au Lac Tanganyika. Bruxelles; Publication de la direction de l'agriculture des forêts et de l'élevage, $103 \mathrm{p}$.

Coulter GW, Martens K, Goddeeris B, 1994. Lake Tanganyika. In: Speciation in Ancient lakes. Archiv Fur Hydrobiologia, $44 \mathrm{p}$.

FAO. 2009. Vue générale du secteur des pêches national République Démocratique du Congo. Profils de pêche et de l'Aquaculture par pays. $F I D / C P / R D C$, FAO 2009, Rome, Italie, $70 \mathrm{p}$.

Kees L, Mambona WB. 1992. Caractéristiques socio-économiques de la pêche congolaise de la partie nord du Lac Tanganyika. Projet régional PNUD/FAO pour la planification, le développement et l'aménagement des pêches continentales en Afrique Orientale/Centrale/Australe. RAF/87/099-TD/37/92, 1-56.

Lindley R. 2000. Etude Spéciale des Pratiques de Pêche (ESPP), Rapport Final. Engins 
de Pêche du Lac Tanganyika au tournant du Millénaire. Lutte contre la pollution et autres mesures visant à protéger la biodiversité du Lac Tanganyika (RAF/92/G32). PARTIE B, Descriptions détaillées des engins, $63 \mathrm{p}$.

Luhusu FK, Micha JC. 2013. Analyse des modes d'exploitation des ressources ha lieutiques du lac Maï-Ndombe en République Démocratique du Congo. Geo-Eco-Trop., 37(2): 273-284.

Mannini P, Aro E, Katonda I, Kassaka B, Mambona C, Milindi G, Paffen P, Verburg P. 1997. Pelagic fish stocks of Lake Tanganyika: Biology and exploitation. Research for the Management of the Fisheries on Lake TanganyikaGCP/RAF/271/FIN-TD/53 (En), $65 \mathrm{p}$.

Mölsä H, Reynolds JE, Coenen EJ, Lindqvist OV. 1999. Fisheries research toward resource management on Lake Tanganyika. Hydrobiologia, 407: 1-24.

Mulimbwa N., 1995. La biologie des poissons pélagiques du Lac Tanganyika.Research for management of the fisheries on Lake Tanganyika. GCP/RAF/271/FIN-TD/53, $10 \mathrm{p}$.

Mushagalusa CD, Micha J-C, Ntakimazi G, Nshombo M. 2015. Brief evolution of the current state of fish stocks landed by artisanal fishing units from the extreme northwest part of Lake Tanganyika. International Journal of Fisheries and Aquatic Studies: 41-48.

Mushagalusa CD, Nshombo M, Lushombo M. 2014. Littoral fisheries on Cichlidae (Pisces) from the northwestern part of Lake Tanganyika, East Africa. Aquatic Ecosystem Health \& Management, 17(1): 41-51.
Njifonjou O. 2010. Méthodologie d'étude de couts et revenus en pêche artisanale, Programme TCP/FAO/CRAF/3101.45 p

PRODAP. 2011. Strategic Action Programme for the Protection of Biodiversity and Sustainable Management of Natural Resources in Lake Tanganyika and its Basin, Bujumbura, Burundi, $141 \mathrm{p}$.

Roest FC. 1978. Stolothrissa tanganicae: population dynamics, biomass evolution and life history in Burundi waters of Lake Tanganyika. CIFA Technical Paper, CPCA 5, 42-46.

Roest FC. 1992. The pelagic fisheries resources of Lake Tanganyika. Mitt Internationalen Verrein Limnologie, 23: 11-15.

Roest FC. 2014. A second Look at the Industrial Fisheries Data, Burundi, Lake Tanagnyika (1973-1992). Bull. Séanc. Acad. R. Sci. Outre-Mer, 60: 351-360.

Sarvala J, Langenberg VT, Solanen K, Chitamwebwa D, Coulter GW, Huttula T, Kanyaru R, Katalainen P, Makassa L, Mulimbwa N, Mölsä H. 2006. Fish catches from Lake Tanganyika mainly reflect changes in fishery practices, not climate. Verhandlungen des Internationalen Verein Limnologie, 29: 1182-1188.

Van der Knaap M. 2013. Comparative analysis of fisheries restoration and public participation in Lake Victoria and Lake Tanganyika.Aquatic Ecosystem Health \& Management, 16(3): 1-9.

Van der Knaap M, Katonda KI, De Graaf GJ. 2014. Lake Tanganyika fisheries frame survey analysis: Assessment of the options for management of the fisheries of Lake Tanganyika. Aquatic Ecosystem Health \& Management, 17(1): 4-13. 